\title{
Chip-Firing And A Devil's Staircase
}

\author{
Lionel Levine \\ Department of Mathematics, Massachusetts Institute of Technology, Cambridge, MA 02139
}

The devil's staircase - a continuous function on the unit interval [0,1] which is not constant, yet is locally constant on an open dense set - is the sort of exotic creature a combinatorialist might never expect to encounter in "real life." We show how a devil's staircase arises from the combinatorial problem of parallel chip-firing on the complete graph. This staircase helps explain a previously observed "mode locking" phenomenon, as well as the surprising tendency of parallel chip-firing to find periodic states of small period.

Keywords: Circle map, fixed-energy sandpile, mode locking, non-ergodicity, parallel chip-firing, rotation number, short period attractors

\section{Introduction}

In this extended abstract, we summarize recent work relating the Poincaré rotation number of a circle map $S^{1} \rightarrow S^{1}$ to the behavior of parallel chip-firing on the complete graph. We use this connection to shed light on two intriguing features of parallel chip-firing, mode locking and short period attractors. Ever since Bagnoli, Cecconi, Flammini, and Vespignani [1] found evidence of mode locking and short period attractors in numerical experiments in 2003 , these two phenomena have called out for a mathematical explanation. The proofs omitted here can be found in [12].

In chip-firing on a finite graph, each vertex $v$ starts with a pile of $\sigma(v) \geq 0$ chips. A vertex is unstable if it has at least as many chips as its degree, and can fire by sending one chip to each neighbor. In parallel chip-firing, at each time step, all unstable vertices fire simultaneously. If it is possible in finitely many firings to reach a stable configuration in which no vertex can fire, then this final configuration does not depend on the order of firings [5]. In this case, the parallel restriction does not affect the final outcome. However, our focus will be on chip configurations that do not stabilize.

Previous work on parallel chip-firing [3, 4, 10, 14] has focused on the periodicity of the dynamics: given a graph $G$, for which natural numbers $q$ does there exist a parallel chip-firing state on $G$ which first recurs after $q$ time steps? We will have more to say about this question below. In the statistical physics literature, parallel chip-firing often goes by the name "fixed energy sandpile" [1, 6, 7, 15]. The term "sandpile" refers to the Bak-Tang-Wiesenfeld model of self-organized criticality [2], while "fixed energy" refers to the lack of a sink or boundary vertex where chips disappear.

We add loops to the complete graph $K_{n}$, so that a vertex with $n$ or more chips is unstable, and fires by sending one chip to each vertex of $K_{n}$, including one chip to itself. The parallel update rule fires all 
unstable vertices simultaneously, yielding a new chip configuration $U \sigma$ given by

$$
U \sigma(v)= \begin{cases}\sigma(v)+r(\sigma), & \sigma(v) \leq n-1 \\ \sigma(v)-n+r(\sigma), & \sigma(v) \geq n\end{cases}
$$

Here

$$
r(\sigma)=\#\{v \mid \sigma(v) \geq n\}
$$

is the number of unstable vertices. Write $U^{0} \sigma=\sigma$, and $U^{t} \sigma=U\left(U^{t-1} \sigma\right)$ for $t \geq 1$.

Note that the total number of chips in the system is conserved. In particular, only finitely many different states are reachable from the initial configuration $\sigma$, so the sequence $\left(U^{t} \sigma\right)_{t \geq 0}$ is eventually periodic: there exist integers $m \geq 1$ and $t_{0} \geq 0$ such that

$$
U^{t+m} \sigma=U^{t} \sigma \quad \forall t \geq t_{0} .
$$

The activity of $\sigma$ is the limit

$$
a(\sigma)=\lim _{t \rightarrow \infty} \frac{\alpha_{t}}{n t}
$$

where

$$
\alpha_{t}=\sum_{s=0}^{t-1} r\left(U^{s} \sigma\right)
$$

is the total number of firings performed in the first $t$ updates. By (2), the limit in (3) exists and equals $\frac{1}{m n}\left(\alpha_{t_{0}+m}-\alpha_{t_{0}}\right)$. Since $0 \leq \alpha_{t} \leq n t$, we have $0 \leq a(\sigma) \leq 1$.

Following [1], we ask: how does the activity change when chips are added to the system? If $\sigma_{n}$ is a chip configuration on $K_{n}$, write $\sigma_{n}+k$ for the configuration obtained from $\sigma_{n}$ by adding $k$ chips at each vertex. The function

$$
\tilde{s}_{n}(k)=a\left(\sigma_{n}+k\right)
$$

is called the activity phase diagram of $\sigma_{n}$. Surprisingly, for many choices of $\sigma_{n}$, the function $\tilde{s}_{n}$ looks like a staircase, with long intervals of constancy punctuated by sudden jumps (Figure 1). This phenomenon is known as mode locking: if the system is in a preferred mode, corresponding to a wide stair in the staircase, then even a relatively large perturbation in the form of adding extra chips will not change the activity. For a general discussion of mode locking in dynamical systems, including examples from astronomy and physics, see [11].

To quantify the idea of mode locking in our setting, suppose we are given an infinite family of chip configurations $\sigma_{2}, \sigma_{3}, \ldots$ with $\sigma_{n}$ defined on $K_{n}$. Suppose $\sigma_{n}$ is stable, i.e.,

$$
0 \leq \sigma_{n}(v) \leq n-1
$$

for all $v \in[n]$. Moreover, suppose that there is a continuous function $F:[0,1] \rightarrow[0,1]$, such that for all $0 \leq x \leq 1$

$$
\frac{1}{n} \#\left\{v \in[n] \mid \sigma_{n}(v)<n x\right\} \rightarrow F(x)
$$

as $n \rightarrow \infty$. Then according to Theorem 3.1 , the activity phase diagrams $\tilde{s}_{n}$, suitably rescaled, converge pointwise to a continuous, nondecreasing function $s:[0,1] \rightarrow[0,1]$. 


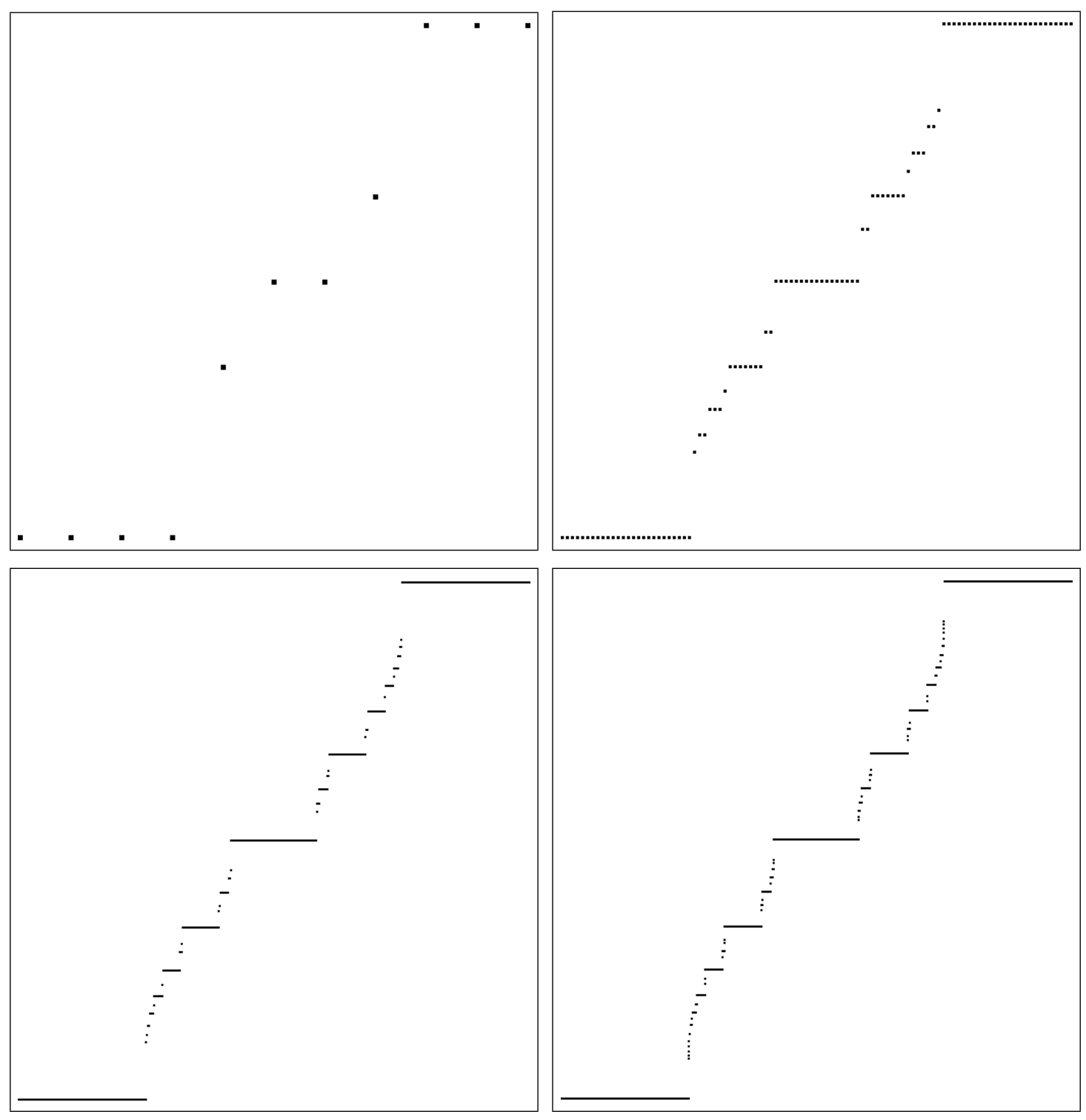

Fig. 1: The activity phase diagrams $a\left(\sigma_{n}+k\right)$, for $n=10$ (top left), 100 (top right), 1000 (bottom left), and 10000, where $\sigma_{n}$ is given by 6 . On the horizontal axis, $k$ runs from 0 to $n$. On the vertical axis, $a\left(\sigma_{n}+k\right)$ runs from 0 to 1 . 
Moreover, under a mild additional hypothesis, Proposition 3.2 says that this limiting function $s$ is a devil's staircase: it is locally constant on an open dense subset of $[0,1]$. For each rational number $p / q \in[0,1]$ there is a stair of height $p / q$, that is, an interval of positive length on which $s$ is constant and equal to $p / q$.

Related to mode locking, a second feature observed in simulations of parallel chip-firing is nonergodicity: in trials performed with random initial configurations on the $n \times n$ torus, the activity observed in individual trials differs markedly from the average activity observed over many trials [15]. The experiments of [1] suggested a reason: the chip-firing states in locked modes, corresponding to stairs of the devil's staircase, tend to be periodic with very small period. We study these short period attractors in Theorem 4.6. Under the same hypotheses that yield a devil's staircase in Propositon 3.2, for each $q \in \mathbb{N}$, a constant fraction $c_{q} n$ of the states $\left\{\sigma_{n}+k\right\}_{k=0}^{n}$ have eventual period $q$.

To illustrate these results, consider the chip configuration $\sigma_{n}$ on $K_{n}$ defined by

$$
\sigma_{n}(v)=\left\lfloor\frac{n}{4}\right\rfloor+\left\lfloor\frac{v-1}{2}\right\rfloor, \quad v=1, \ldots, n .
$$

Here $\lfloor x\rfloor$ denotes the greatest integer $\leq x$. This family of chip configurations satisfies (??) with

$$
F(x)= \begin{cases}0, & x \leq \frac{1}{4} \\ 2 x-\frac{1}{2}, & \frac{1}{4} \leq x \leq \frac{3}{4} \\ 1, & x \geq \frac{3}{4}\end{cases}
$$

The activity phase diagrams of $\sigma_{n}$ for $n=10,100,1000,10000$ are graphed in Figure 1 For example, when $n=10$ we have

$$
\left(a\left(\sigma_{10}+k\right)\right)_{k=0}^{10}=(0,0,0,0,1 / 3,1 / 2,1 / 2,2 / 3,1,1,1)
$$

and when $n=100$, we have

$\left(a\left(\sigma_{100}+k\right)\right)_{k=0}^{100}=(0,0,0,0,0,0,0,0,0,0,0,0,0,0,0,0,0,0,0,0,0,0,0,0,0,0,1 / 6,1 / 5,1 / 5,1 / 4$, $1 / 4,1 / 4,2 / 7,1 / 3,1 / 3,1 / 3,1 / 3,1 / 3,1 / 3,1 / 3,2 / 5,2 / 5,1 / 2,1 / 2,1 / 2,1 / 2,1 / 2,1 / 2,1 / 2,1 / 2,1 / 2,1 / 2,1 / 2$, $1 / 2,1 / 2,1 / 2,1 / 2,1 / 2,1 / 2,3 / 5,3 / 5,2 / 3,2 / 3,2 / 3,2 / 3,2 / 3,2 / 3,2 / 3,5 / 7,3 / 4,3 / 4,3 / 4,4 / 5,4 / 5,5 / 6,1,1,1$, $1,1,1,1,1,1,1,1,1,1,1,1,1,1,1,1,1,1,1,1,1,1,1)$.

As $n$ grows, the denominators of these rational numbers grow remarkably slowly: the largest denominator is 11 for $n=1000$, and 13 for $n=10000$. Moreover, for any fixed $n$ the very smallest denominators occur with greatest frequency. For example, when $n=10000$, there are 1667 values of $k$ for which $a\left(\sigma_{n}+k\right)=\frac{1}{2}$, and 714 values of $k$ for which $a\left(\sigma_{n}+k\right)=\frac{1}{3}$; but for each $p=1, \ldots, 12$ there is just one value of $k$ for which $a\left(\sigma_{n}+k\right)=\frac{p}{13}$. In Lemma 4.5, we relate these denominators to the periodicity: if $a(\sigma)=p / q$ in lowest terms, then $\sigma$ has eventual period $q$.

The remainder of the paper is organized as follows. In section 2 we show how to construct, given a chip configuration $\sigma$ on $K_{n}$, a circle map $f: S^{1} \rightarrow S^{1}$ whose rotation number equals the activity of $\sigma$. This construction resembles the one-dimensional particle/barrier model of [9]. In section 3 we use the circle map to prove our main results on mode locking, Theorem 3.1 and Proposition 3.2 Short period attractors are studied in section 4 , where we show that all states on $K_{n}$ have eventual period at most $n$ 
(Proposition 4.4). Finally, in Theorem 4.11, we find a small "window" in which all states have eventual period two.

Many questions remain concerning parallel chip-firing on graphs other than $K_{n}$. If the underlying graph is a tree [4] or a cycle [7], then instead of a devil's staircase of infinitely many preferred modes, there are just three: activity $0, \frac{1}{2}$ and 1 . On the other hand, the numerical experiments of [1] for parallel chip-firing on the $n \times n$ torus suggest a devil's staircase in the large $n$ limit. Our arguments rely quite strongly on the structure of the complete graph, whereas the mode locking phenomenon seems to be widespread. It would be very interesting to relate parallel chip-firing on other graphs to iteration of a circle map (or perhaps a map on a higher-dimensional manifold) in order to explain the ubiquity of mode locking.

\section{Construction of the Circle Map}

We first identify a certain class of chip configurations on $K_{n}$, the confined states, with the property that for any configuration $\sigma$ of activity $a(\sigma)<1$, we have $U^{t} \sigma$ confined for all sufficiently large $t$.

Definition. A chip configuration $\sigma$ on $K_{n}$ is preconfined if it satisfies

(i) $\sigma(v) \leq 2 n-1$ for all vertices $v$ of $K_{n}$.

If, in addition, $\sigma$ satisfies

(ii) $\max _{v} \sigma(v)-\min _{v} \sigma(v) \leq n-1$

then $\sigma$ is confined.

Lemma 2.1. If $\sigma$ is preconfined, then $U \sigma$ is confined.

Lemma 2.2. If $a(\sigma)<1$, then $U^{t} \sigma$ is confined for all sufficiently large $t$.

Note that from (1)

$$
U \sigma(v) \equiv \sigma(v)+r(\sigma) \quad(\bmod n)
$$

Iterating yields the congruence

$$
U^{t} \sigma(v) \equiv \sigma(v)+\alpha_{t} \quad(\bmod n)
$$

where

$$
\alpha_{t}=\sum_{s=0}^{t-1} r\left(U^{s} \sigma\right)
$$

is the total number of firings before time $t$.

Our next lemma characterizes the vertices that fire at time $t+1$.

Lemma 2.3. If $U^{t} \sigma$ is preconfined, then $U^{t+1} \sigma(v) \geq n$ if and only if

$$
\sigma(v) \equiv-j(\bmod n)
$$

for some $\alpha_{t}<j \leq \alpha_{t+1}$. 
Let

$$
\phi(j)=\#\{v \in[n] \mid \sigma(v) \equiv-j(\bmod n)\} .
$$

By Lemma 2.3, if $U^{t} \sigma$ is preconfined, then the number of unstable vertices in $U^{t+1} \sigma$ is

$$
r_{t+1}=\phi\left(\alpha_{t}+1\right)+\ldots+\phi\left(\alpha_{t+1}\right),
$$

hence

$$
\alpha_{t+2}=\alpha_{t+1}+\sum_{j=\alpha_{t}+1}^{\alpha_{t+1}} \phi(j) .
$$

This gives a recurrence for $\alpha_{t}$ relating three consecutive terms $\alpha_{t}, \alpha_{t+1}$ and $\alpha_{t+2}$. Our next lemma simplifies this to a recurrence relating just two consecutive terms.

Lemma 2.4. If $\sigma$ is preconfined, then for all $t \geq 0$

$$
\alpha_{t+1}=g\left(\alpha_{t}\right),
$$

where $g: \mathbb{N} \rightarrow \mathbb{N}$ is given by

$$
g(k)=\alpha_{1}+\sum_{j=1}^{k} \phi(j)
$$

and $\phi$ is given by (9).

The function $g$ appearing in Lemma 2.4 satisfies

$$
\begin{aligned}
g(k+n) & =g(k)+\sum_{j=k+1}^{k+n} \phi(j) \\
& =g(k)+\sum_{j=k+1}^{k+n} \#\{v \mid \sigma(v) \equiv-j(\bmod n)\} \\
& =g(k)+n .
\end{aligned}
$$

for all $k \in \mathbb{N}$. This suggests that a more natural domain of definition is the unit circle. First extend $g$ to all of $\mathbb{Z}$ by defining

$$
g(-k)=g(n k-k)-n k, \quad k \in \mathbb{N} .
$$

This is the unique extension with the property that $g-I d$ is periodic $\bmod n$. Now for $x \in \mathbb{R}$, let

$$
f(x)=\frac{(1-\{n x\}) g(\lfloor n x\rfloor)+\{n x\} g(\lceil n x\rceil)}{n}
$$

where $\lfloor y\rfloor,\lceil y\rceil$ and $\{y\}$ denote respectively the greatest integer $\leq y$, the least integer $\geq y$, and the fractional part of $y$.

By (12) we have for all $x \in \mathbb{R}$

$$
\begin{aligned}
f(x+1) & =\frac{(1-\{n x\}) g(\lfloor n x\rfloor+n)+\{n x\} g(\lceil n x\rceil+n)}{n} \\
& =f(x)+1
\end{aligned}
$$


Hence $f: \mathbb{R} \rightarrow \mathbb{R}$ descends to a circle map $\bar{f}: S^{1} \rightarrow S^{1}$ (viewing $S^{1}$ as $\mathbb{R} / \mathbb{Z}$ ). Since $f$ is nondecreasing, it has a well-defined Poincaré rotation number [8, 13]

$$
\rho(f)=\lim _{t \rightarrow \infty} \frac{f^{t}(x)}{t}
$$

which does not depend on $x$. Here $f^{t}$ denotes the $t$-fold iterate $f^{t}(x)=f\left(f^{t-1}(x)\right)$, with $f^{0}=I d$. The rotation number measures the rate at which the sequence of points $x, \bar{f}(x), \bar{f}(\bar{f}(x)), \ldots$ winds around the circle.

Theorem 2.5. If $\sigma$ is preconfined, then $a(\sigma)=\rho(f)$.

Note that the map $g$ is defined in terms of $\alpha_{1}$ and $\phi$, both of which are easily read off from $\sigma$. So given a preconfined configuration $\sigma$, equations (11) and (13) give an explicit recipe for writing down a circle map $f$ whose rotation number is the activity of $\sigma$.

One naturally wonders how to generalize this construction to chip-firing configurations on graphs other than $K_{n}$. A key step may involve identifying invariants of the dynamics. On $K_{n}$, these invariants take a very simple form: by $[8$, for any two vertices $v, w \in[n]$, the difference

$$
U^{t} \sigma(v)-U^{t} \sigma(w) \bmod n
$$

does not depend on $t$. Analogous invariants for parallel chip-firing on the $n \times n$ torus are classified in [6].

\section{Devil's Staircase}

Let $\sigma_{2}, \sigma_{3}, \ldots$ be a sequence of chip configurations, with $\sigma_{n}$ defined on $K_{n}$, satisfying the conditions (4) and (5). Extend $F$ to all of $\mathbb{R}$ by setting

$$
F(x+m)=F(x)+m, \quad m \in \mathbb{Z} .
$$

Note that (4) and 5 force $F(0)=0$ and $F(1)=1$, so this extension is continuous.

The rescaled activity phase diagram of $\sigma_{n}$ is the function $s_{n}:[0,1] \rightarrow[0,1]$ defined by

$$
s_{n}(y)=a\left(\sigma_{n}+\lfloor n y\rfloor\right) .
$$

As $n \rightarrow \infty$, the $s_{n}$ have a pointwise limit identified in our next result. Here and in what follows, $\rho(\cdot)$ denotes the rotation number (14).

Theorem 3.1. If (4) and (5) hold, then for each $y \in[0,1]$ we have

$$
s_{n}(y) \rightarrow s(y):=\rho\left(R_{y} \circ \Phi\right)
$$

as $n \rightarrow \infty$, where $\Phi(x)=-F(-x)$, and $R_{y}(x)=x+y$.

Write $\Phi_{y}=R_{y} \circ \Phi$, and let $\bar{\Phi}_{y}: S^{1} \rightarrow S^{1}$ be the corresponding circle map. We will call a function $s:[0,1] \rightarrow[0,1]$ a devil's staircase if it is continuous, nondecreasing, nonconstant, and locally constant on an open dense set. Next we show that if

$$
\left(\bar{\Phi}_{y}\right)^{q} \neq I d \quad \text { for all } y \in S^{1} \text { and all } q \in \mathbb{N},
$$

then the limiting function $s(y)$ in Theorem 3.1 is a devil's staircase. Examples of these staircases for different choices of $F$ are shown in Figure 2 . 


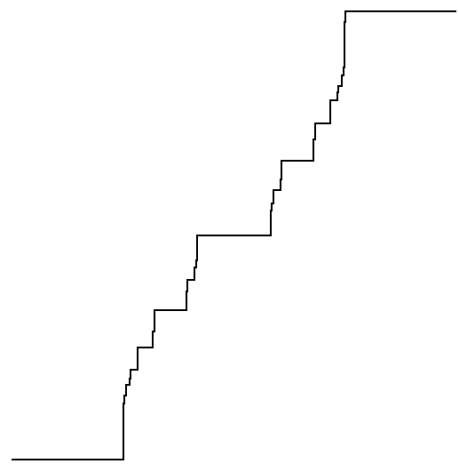

(a)

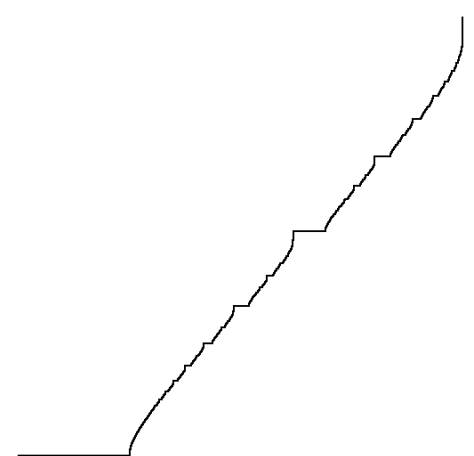

(b)

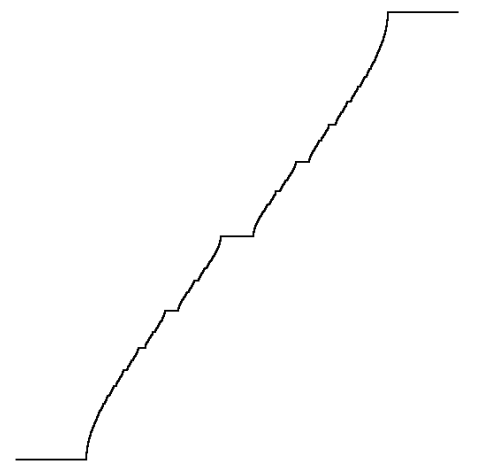

(c)

Fig. 2: The devil's staircase $s(y)$, when (a) $F(x)$ is given by 77 ; (b) $F(x)=\sqrt{x}$ for $x \in[0,1]$; and (c) $F(x)=$ $x+\frac{1}{2 \pi} \sin 2 \pi x$. On the horizontal axis $y$ runs from 0 to 1 , and on the vertical axis $s(y)$ runs from 0 to 1 .

Proposition 3.2. The function $s(y)=\rho\left(\Phi_{y}\right)$ continuous and nondecreasing in $y$. If $z \in[0,1]$ is irrational, then $s^{-1}(z)$ is a point. Moreover, if (16) holds, then for every rational number $p / q \in[0,1]$ the fiber $s^{-1}(p / q)$ is an interval of positive length.

Our next result shows that in the interiors of the stairs, we in fact have $s_{n}(y)=s(y)$ for sufficiently large $n$.

Proposition 3.3. Suppose that (4), (5) and (16) hold. If $s^{-1}(p / q)=[a, b]$, then for any $\epsilon>0$

$$
[a+\epsilon, b-\epsilon] \subset s_{n}^{-1}(p / q)
$$

for all sufficiently large $n$.

The results in this section follow from Theorem 2.5 along with a few well-known properties of the rotation number $\rho(f)$. To give a flavor of the proofs, we list here the properties we use. For more background on the rotation number, see [8, 13].

Call a map $f: \mathbb{R} \rightarrow \mathbb{R}$ a monotone degree one lift if $f$ is continuous, nondecreasing and satisfies

$$
f(x+1)=f(x)+1
$$

for all $x \in \mathbb{R}$. Let $f, f_{n}, g$ be monotone degree one lifts, and denote by $\bar{f}, \bar{f}_{n}, \bar{g}$ the corresponding circle maps $S^{1} \rightarrow S^{1}$. Write $f \leq g$ if $f(x) \leq g(x)$ for all $x \in \mathbb{R}$, and $f<g$ if $f(x)<g(x)$ for all $x \in \mathbb{R}$.

- Monotonicity. If $f \leq g$, then $\rho(f) \leq \rho(g)$.

- Continuity. If $\sup \left|f_{n}-f\right| \rightarrow 0$, then $\rho\left(f_{n}\right) \rightarrow \rho(f)$.

- Conjugation Invariance. If $g$ is strictly increasing, then $\rho\left(g \circ f \circ g^{-1}\right)=\rho(f)$.

- Instability of an irrational rotation number. If $\rho(f) \notin \mathbb{Q}$, and $f_{1}<f<f_{2}$, then

$$
\rho\left(f_{1}\right)<\rho(f)<\rho\left(f_{2}\right) .
$$


- Stability of a rational rotation number. If $\rho(f)=p / q \in \mathbb{Q}$, and $\bar{f}^{q} \neq I d: S^{1} \rightarrow S^{1}$, then for sufficiently small $\epsilon>0$, either

$$
\rho(g)=p / q \text { whenever } f \leq g \leq f+\epsilon,
$$

or

$$
\rho(g)=p / q \text { whenever } f-\epsilon \leq g \leq f .
$$

\section{Short Period Attractors}

For a chip configuration $\sigma$ on $K_{n}$ and a vertex $v \in[n]$, let

$$
u_{t}(\sigma, v)=\#\left\{0 \leq s<t \mid U^{s} \sigma(v) \geq n\right\}
$$

be the number of times $v$ fires during the first $t$ updates. During these updates, the vertex $v$ emits a total of $n u_{t}(\sigma, v)$ chips and receives a total of $\alpha_{t}=\sum_{w} u_{t}(\sigma, w)$ chips, so that

$$
U^{t} \sigma(v)-\sigma(v)=\alpha_{t}-n u_{t}(\sigma, v) .
$$

An easy consequence is the following.

Lemma 4.1. A chip configuration $\sigma$ on $K_{n}$ satisfies $U^{t} \sigma=\sigma$ if and only if

$$
u_{t}(\sigma, v)=u_{t}(\sigma, w)
$$

for all vertices $v$ and $w$.

According to our next lemma, if $\sigma$ is confined, then $u_{t}(\sigma, v)$ and $u_{t}(\sigma, w)$ differ by at most one.

Lemma 4.2. If $\sigma$ is confined, and $\sigma(v) \leq \sigma(w)$, then for all $t \geq 0$

$$
u_{t}(\sigma, v) \leq u_{t}(\sigma, w) \leq u_{t}(\sigma, v)+1 .
$$

Lemma 4.3. If $\sigma$ is confined, then $U^{t} \sigma=\sigma$ if and only if $n \mid \alpha_{t}$.

Let $\sigma$ be a confined state on $K_{n}$. By the pigeonhole principle, there exist times $0 \leq s<t \leq n$ with

$$
\alpha_{s} \equiv \alpha_{t} \quad(\bmod n) .
$$

By Lemma 4.3 it follows that $U^{s} \sigma=U^{t} \sigma$, so $\sigma$ has eventual period at most $n$.

Our next result improves this bound a bit. Write $m(\sigma)$ for the eventual period of $\sigma$, and

$$
\nu(\sigma)=\#\{\sigma(v) \mid v \in[n]\}
$$

for the number of distinct heights in $\sigma$.

Proposition 4.4. For any chip configuration $\sigma$ on $K_{n}$,

$$
m(\sigma) \leq \nu(\sigma) .
$$


Bitar [3] conjectured that any parallel chip-firing configuration on a connected graph of $n$ vertices has eventual period at most $n$. A counterexample was found by Kiwi et al. [10]. It would be interesting to investigate for what classes of graphs Bitar's conjecture does hold; for example, no counterexample seems to be known on a regular graph.

Next we relate the period to the activity.

Lemma 4.5. If $a(\sigma)=p / q$ and $(p, q)=1$, then $m(\sigma)=q$.

The proof uses the fact that the rotation number of a circle map determines the periods of its periodic points: if $f: \mathbb{R} \rightarrow \mathbb{R}$ is a monotone degree one lift (17) with $\rho(f)=p / q$ in lowest terms, then all periodic points of $\bar{f}: S^{1} \rightarrow S^{1}$ have period $q$; see Proposition 4.3.8 and Exercise 4.3.5 of [8].

Given $1 \leq p<q \leq n$ with $(p, q)=1$ and $p / q \leq 1 / 2$, one can check that the chip configuration $\sigma$ on $K_{n}$ given by

$$
\sigma(v)= \begin{cases}v+p-1, & v \leq q-1-p \\ v+n+p-q-1, & q-p \leq v \leq q-1 \\ n+p-1, & v \geq q .\end{cases}
$$

has activity $a(\sigma)=p / q$. For a similar construction on more general graphs in the case $p=1$, see [14, Prop. 6.8]. In particular, $m(\sigma)=q$ by Lemma 4.5. So for every integer $q=1, \ldots, n$ there exists a chip configuration on $K_{n}$ of period $q$.

Despite the existence of states of period as large as $n$, states of smaller period are in some sense more prevalent. One way to capture this is the following.

Theorem 4.6. If $\sigma_{2}, \sigma_{3}, \ldots$ is a sequence of chip configurations satisfying (4), (5) and (16), then for each $q \in \mathbb{N}$ there is a constant $c=c_{q}>0$ such that for all sufficiently large $n$, at least cn of the states $\left\{\sigma_{n}+k\right\}_{k=0}^{n}$ have eventual period $q$.

The proof follows easily from Proposition 3.3 , which shows that a constant fraction $c n$ of the states $\sigma_{n}+k$ have activity $1 / q$. By Lemma 4.5 these states have eventual period $q$. The devil's staircase $s(y)$ determines the best possible constant $c_{q}$, namely, the total length of all stairs whose height has denominator $q$. If $s^{-1}(p / q)=\left[a_{p}, b_{p}\right]$, then any constant

$$
c_{q}<\sum_{p:(p, q)=1}\left(b_{p}-a_{p}\right)
$$

satisfies the conclusion of the theorem.

The rest of this section outlines the proof of Theorem 4.11, which finds a period 2 window: any chip configuration on $K_{n}$ with total number of chips strictly between $n^{2}-n$ and $n^{2}$ has eventual period 2 . The following lemma is a special case of [14, Prop. 6.2].

Lemma 4.7. If $\sigma$ and $\tau$ are chip configurations on $K_{n}$ with $\sigma(v)+\tau(v)=2 n-1$ for all $v$, then $a(\sigma)+a(\tau)=1$.

Given a chip configuration $\sigma$ on $K_{n}$, for $j=1, \ldots, n$ we define conjugate configurations

$$
c^{j} \sigma(v)= \begin{cases}\sigma(v)+j-n, & v \leq j \\ \sigma(v)+j, & v>j .\end{cases}
$$


Lemma 4.8. Let $\sigma$ be a chip configuration on $K_{n}$, and fix $j \in[n]$. For all $t \geq 0$, we have for $v \leq j$

$$
u_{t}(\sigma, v)-1 \leq u_{t}\left(c^{j} \sigma, v\right) \leq u_{t}(\sigma, v)
$$

while for $v>j$

$$
u_{t}(\sigma, v) \leq u_{t}\left(c^{j} \sigma, v\right) \leq u_{t}(\sigma, v)+1
$$

Corollary 4.9. For any chip configuration $\sigma$ on $K_{n}$ and any $j \in[n]$,

$$
a\left(c^{j} \sigma\right)=a(\sigma)
$$

It turns out that the circle maps corresponding to $\sigma$ and $c^{j} \sigma$ are conjugate to one another by a rotation. This gives an alternative proof of the corollary, in the case when both $\sigma$ and $c^{j} \sigma$ are preconfined.

Lemma 4.10. Let $\sigma$ be a chip configuration on $K_{n}$. If $u_{2}(\sigma, v) \geq 1$ for all $v$, then $u_{2 t}(\sigma, v) \geq t$ for all $v$ and all $t \geq 1$.

Write

$$
|\sigma|=\sum_{v=1}^{n} \sigma(v)
$$

for the total number of chips in the system.

Theorem 4.11. Every chip configuration $\sigma$ on $K_{n}$ with $n^{2}-n<|\sigma|<n^{2}$ has eventual period 2.

The outline of the proof runs as follows. Writing

$$
\ell(\sigma)=\min \{\sigma(1), \ldots, \sigma(n)\}
$$

and

$$
r(\sigma)=\#\{v \in[n]: \sigma(v) \geq n\},
$$

a straightforward calculation shows that if $\sigma(1) \geq \sigma(2) \geq \ldots \geq \sigma(n)$ and $n^{2}-n<|\sigma|<n^{2}$, then

$$
\sum_{j=1}^{n}\left(\ell\left(c^{j} \sigma\right)+r\left(c^{j} \sigma\right)\right)>n^{2}-n .
$$

Since each term in the sum on the left is a nonnegative integer, we must have

$$
\ell\left(c^{j} \sigma\right)+r\left(c^{j} \sigma\right) \geq n
$$

for some $j \in[n]$. Thus every vertex $v$ fires at least once during the first two updates of $c^{j} \sigma$. By Corollary 4.9 and Lemma 4.10, this implies

$$
a(\sigma)=a\left(c^{j} \sigma\right) \geq \frac{1}{2}
$$

The chip configuration $\tau(v):=2 n-1-\sigma(v)$ also satisfies $n^{2}-n<|\tau|<n^{2}$, so $a(\tau) \geq \frac{1}{2}$ as well. By Lemma 4.7 we have $a(\sigma)+a(\tau)=1$, so $a(\sigma)=a(\tau)=\frac{1}{2}$. Finally, from Lemma 4.5 we conclude that $m(\sigma)=2$. 


\section{Acknowledgement}

The author thanks Anne Fey for many helpful conversations.

\section{References}

[1] F. Bagnoli, F. Cecconi, A. Flammini, and A. Vespignani, Short-period attractors and non-ergodic behavior in the deterministic fixed-energy sandpile model, Europhys. Lett. 63 (2003), 512-518.

[2] P. Bak, C. Tang and K. Wiesenfeld, Self-organized criticality: an explanation of the $1 / f$ noise, Phys. Rev. Lett. 59, no. 4 (1987), 381-384.

[3] J. Bitar, Juegos Combinatoriales en Redes de Autómatas, Tesis Ingeniero Matemático, Dept. Ingeniería Matemática, U. de Chile, 1989.

[4] J. Bitar and E. Goles, Parallel chip firing games on graphs, Theoretical Comp. Sci. 92, no. 2 (1992), 291-300.

[5] A. Björner, L. Lovász and P. Shor, Chip-firing games on graphs, European J. Combin. 12 (1991), no. 4, 283-291.

[6] M. Casartelli, L. Dall'Asta, A. Vezzani and P. Vivo, Dynamical invariants in the deterministic fixedenergy sandpile, European Phys. J. B 52 (2006), no. 1, 91-105.

[7] L. Dall'Asta, Exact solution of the one-dimensional deterministic fixed-energy sandpile, Phys. Rev. Lett. 96 (2006).

[8] B. Hasselblatt and A. Katok, A First Course in Dynamics, With a Panorama of Recent Developments, Cambridge University Press, 2003.

[9] S. A. Janowsky and C. A. Laberge, Exact solutions for a mean-field abelian sandpile, J. Phys. A 26 (1993) L973-L980.

[10] M. A. Kiwi, R. Ndoundam, M. Tchuente and E. Goles, No polynomial bound for the period of the parallel chip firing game on graphs, Theoretical Comp. Sci. 136, no. 2 (1994), 527-532.

[11] J. C. Lagarias, Number theory and dynamical systems, Proc. Symp. Applied Math. 46 (1992), $35-72$.

[12] L. Levine, Parallel chip-firing on the complete graph: devil's staircase and Poincaré rotation number, http://arxiv.org/abs/0811.2800.

[13] W. de Melo and S. van Strien, One-Dimensional Dynamics, Springer-Verlag, 1991.

[14] E. Prisner, Parallel chip-firing on digraphs, Complex Systems 8 (1994), 367-383.

[15] A. Vespignani, R. Dickman, M. A. Muñoz, and S. Zapperi, Absorbing-state phase transitions in fixed-energy sandpiles, Phys. Rev. E 62 (2000), 4564-4582. 